\title{
Factores de riesgo social en el desempeño escolar
}

\author{
Elisa Piedra ${ }^{1}$, Ximena Vélez ${ }^{1}$, Liliana Arciniegas ${ }^{1}$, Ana Pacurucu ${ }^{2}$, Patricio Cabrera ${ }^{1}$, Franklin \\ Mora $^{I}$ \\ ${ }^{1}$ Escuela de Educación Especial, Facultad de Filosofía, Universidad del Azuay, Cuenca, Ecuador. \\ ${ }^{2}$ Escuela de Psicología, Facultad de Filosofía, Universidad del Azuay, Cuenca, Ecuador. \\ Autor para correspondencia: epiedra@uazuay.edu.ec
}

Fecha de recepción: 1 de agosto 2013 - Fecha de aceptación: 22 de enero 2014

\begin{abstract}
RESUMEN
El presente artículo explora algunos de los factores de riesgo responsables de bajo rendimiento escolar en niños entre el 4to a 6to año de educación básica en las escuelas primarias públicas de la ciudad de Cuenca, Ecuador. Para este fin, se entrevistó a los padres, maestros, directores de escuela y niños con rendimiento académico alto y bajo, respectivamente, utilizando una plantilla en la que se evaluaron las características de la población, las condiciones socioeconómicas, la actitud de los niños y los maestros, y las variables de rendimiento. Para este estudio se realizaron un total de 786 encuestas, en diferentes escuelas primarias públicas de la ciudad de Cuenca, de las cuales 77 fueron eliminados por la información incompleta. Los resultados mostraron que el bajo rendimiento escolar de los niños en primer lugar se debe a problemas de salud, la desnutrición, la alta rotación de los docentes, la situación socioeconómica de los padres, y el grado de repetición de años escolares. Además, se demostró que los niños en general tienen una puntuación inferior a las niñas.
\end{abstract}

Palabras clave: Escuela básica pública, bajo rendimiento escolar, actores educativos, riesgo social.

\begin{abstract}
This paper explores some of the risk factors responsible for low school performance of the 4th to 6th level children of public elementary schools in the city of Cuenca, Ecuador. To this end, children with respectively low and high academic performance, parents, teachers and school directors were interviewed, using a template from which the population characteristics, the socioeconomic conditions, attitude of children and teachers, and performance variables were evaluated. For this study a total of 786 surveys, in different public elementary schools in the city of Cuenca were conducted, of which 77 were eliminated by incompleteness. The results showed that the low school performance of the children in first instance is caused by health problems, malnutrition, the high rotation of teachers, the socioeconomic situation of the parents, and the grade of repetition of school years. Furthermore, it was shown that boys overall score less than girls.
\end{abstract}

Keywords: Public elementary school, low school performance, educational actors, social risks.

\section{INTRODUCCIÓN}

El término riesgo social abarca una diversidad de aspectos que van desde la condición biológica de una persona hasta la situación política de una sociedad. Sin embargo, al estudiar el riesgo social es necesario seleccionar factores de riesgo relativos al contexto en el que se desenvuelve la población. El presente estudio ha seleccionado aspectos directamente relacionados con el campo educativo del Azuay, a saber: factores familiares, escolares, sociales y culturales. Sólo al conocer los factores de riesgo, la realidad es susceptible de intervención. Estudios en torno a los factores de riesgo en el campo educativo, los han definido como rasgos individuales, experiencias o factores ambientales que 
aumentan la probabilidad de resultados negativos (Bowen y col., 2007). Que un estudiante esté en riesgo no significa que sea retrasado o que tenga alguna incapacidad, sino mas bien, se refiere a características de su medio familiar, escolar y social que lo predisponen a experiencias negativas, tales como bajo rendimiento, deserción, trastornos conductuales y problemas emocionales (Jadue, 1997).

En el Ecuador, el 9\% de la población de más de 15 años que asiste regularmente a un centro de estudios aún no ha terminado la educación básica (rezago estudiantil) (INEC, 2011a). La tasa de analfabetismo a nivel nacional es de 6,8\% y en la provincia del Azuay es de 6,7\% y el promedio de años de escolaridad para personas de 24 años y más es de 11,4 años en la zona urbana y 6,5 años en la zona rural. El sistema de la Educación General Básica (EGB) abarca al 95,2\% de los niños con edades entre los 5-14 años (INEC, 2011b). De manera particular en la zona urbana del cantón Cuenca, existen características muy positivas en relación a la calidad de educación y asistencia escolar, pues se dispone de infraestructura y personal docente completo para atender a una población de 41.181 niños cuya edad oscila entre los 6 a 12 años. Al respecto, la educación pública abastece a toda la clase baja y gran parte de la clase media de la ciudad, incluyendo en este conjunto, al 59,23\% de niños de la edad señalada (INEC, 2013).

Aunque se desconocen cifras de la prevalencia de Riesgo Social, los efectos de este fenómeno, en los peores casos, son coadyuvantes de la existencia de un total de 18 millones de niños menores de 14 años que trabajan en lugar de estudiar en Latinoamérica (Kliksberg, 2009). Ello se responsabiliza a problemas de diversa índole según estudios especializados. Uno de ellos es la participación familiar, la cual constituye un factor de riesgo pues se demuestra que la falta de involucramiento de los padres de familia en la EGB está asociada con la deserción escolar. Así Barnard (2004) concluye que esta variable está asociada significativamente con los logros de los niños en la primaria temprana. Además, se tiene evidencias de que este aspecto ocurre frecuentemente en hogares de limitados ingresos económicos (Bonal, 2007). En Ghana, un estudio sobre deserción escolar demostró que en las escuelas públicas existen posicionamientos sociales que afectan sobremanera a los más pobres, pues éstos son marginados por aquellos que no son tan pobres (Dunne y Ananga, 2013). El bajo ingreso per cápita de los padres es otro factor de bajo rendimiento (Zeitlin y col., 1990).

El informe del Banco Interamericano del Desarrollo de 1998 señala que un trabajador que ha alcanzado seis años de educación logra en su primer empleo un ingreso por hora $50 \%$ más elevado que quien no ha asistido a la escuela. Si la escolarización ha sido de 12 años, la distancia se amplía al $120 \%$ y si ha sido de 17 años, supera el 200\%. Estos datos promedio esconden diferencias mayores en algunos países. En Brasil, los trabajadores con seis años de educación reciben casi el doble de ingresos que quienes no han asistido a la escuela; con 12 años, el 170\% y con 17 años, el 280\% (Marchesi, 2002); por lo que se establece una relación dependiente entre la educación y la desigualdad social. El efecto más evidente de la deserción escolar es el trabajo infantil, principalmente de vendedores en las calles o la mendicidad que lejos de estimular la economía genera efectos negativos en el desarrollo cognitivo, emocional y social (Holgado y col., 2014). Por otro lado, comparaciones internacionales han demostrado que quienes participan del crimen, la pobreza y desempleo se caracterizan por tener bajos niveles de educación (Bonal, 2007).

Existen otros factores de riesgo, por ejemplo, la alimentación es crucial en el rendimiento. En Chile una investigación reveló un impacto potencial de la alimentación escolar en los resultados de la educación. Hay tres vías por las que las comidas influyen en el aprendizaje: inducir a las familias a inscribir a sus niños en la escuela y a fomentar una asistencia regular, aumentando incluso las tasas de matrícula (McEwan, 2012).

El Plan Decenal de Educación, plantea la Universalización de la Escolarización de Primero a Décimo de Básica (MINEDUC, 2006). En este Plan, se habla de las acciones a tomar para el logro de esta política: eliminación del aporte voluntario, dotación de textos escolares, aumento en la matriculación en el primero de básica en los regímenes Sierra y Costa. Aunque ello supone un avance en este campo, no se consideran todas las causas y relaciones del desempeño escolar que abarca el riesgo social para garantizar un horizonte de un mínimo de diez años de escolarización y, por lo tanto, promover cambios profundos en la sociedad. En Nigeria, se encontró que la gratuidad era un factor que ayudaba a que los niños de escasos recursos económicos asistan a la escuela, pues al sacar el costo de la educación por niño, la economía de los más pobres no podía cubrirla (Licove, 2009). Es necesario tener en cuenta que los programas de expansión y mejoramiento de la educación en 
Latinoamérica han beneficiado a las clases bajas, pero no a la pobreza extrema, debido a que se requiere un mínimo de ingresos para participar de la educación, lo cual ha llevado a que se planteen nuevos objetivos en los que la prioridad sea la educación básica y no simplemente el acceso a la educación (Bonal, 2007). En Brasil, el conocimiento de los factores de riesgo han permitido diseñar políticas para que las familias se acojan a ellas y puedan reaccionar de mejor manera ante las diferentes amenazas que tienen los niños en las escuelas urbanas (da Cunha y col., 2009).

Frente a este contexto, es que la presente investigación plantea establecer relaciones entre estos tres elementos: el desempeño escolar, su relación con el riesgo social y la participación de los actores educativos como entes de riesgo o protección; determinando, de este modo, que un grupo de niños encuentre buenas razones para abandonar la escuela (González González, 2005). Por su parte, el rendimiento académico de los niños comprende las calificaciones promedio obtenidas en un año lectivo, mismas que se distribuyen en tres grupos, a saber: rendimiento alto, bajo y medio; lo cual permitió contrastar a los que tenían alto y bajo para saber qué factores de riesgo inciden significativamente en su desempeño en la escuela.

Se estableció como objetivo del presente trabajo medir los factores de riesgo que ocasionan en el niño un bajo rendimiento escolar y los factores de protección que influyen en un buen rendimiento escolar en el contexto cuencano. El presente trabajo halla su justificación en el hecho de que muchos docentes consideran a la pobreza como un condicionante de la asistencia, permanencia, calificaciones, repitencia y otros factores que identificarían el desempeño escolar de los niños en la educación básica, sin tomar en cuenta particularidades contextuales de sus estudiantes. Al momento, no existen documentos que se hayan elaborado sistemática y rigurosamente, para conocer sus cifras y relaciones, es decir, bajo qué condiciones se produce o qué hace el sistema educativo en general y los centros escolares para afrontar la relación de riesgo social y desempeño escolar.

\section{MATERIALES Y MÉTODOS}

\subsection{Materiales}

Existe un total de 54 establecimientos públicos en la zona urbana del cantón Cuenca, los cuales abarcan a toda la clase baja y parte de la clase media de la ciudad. La muestra obtenida partió de un universo constituido por todas las escuelas fiscales de la ciudad de Cuenca. Se determinó el tamaño mínimo aconsejado de la muestra para la población de 30.700 escolares de 725 casos. Sin embargo, la muestra incluyó 786 casos, completándose así la muestra requerida. Se realizó un sorteo para escoger las escuelas de forma aleatoria en donde se realizarían las encuestas. Las escuelas fueron: Escuela Fiscal (EF) 3 de noviembre, EF Benigno Astudillo, EF Juan Montalvo, EF Fe y Alegría, EF Cazadores de Los Ríos, EF Manuela Cañizares, EF Remigio Romero y Cordero, EF La Inmaculada y EF Ignacio Escandón. En estos establecimientos, durante el período diciembre 2011-abril 2012, se realizaron 786 encuestas en las escuelas, de las cuales se eliminaron 77 registros por falta de todos los datos.

La muestra de alumnos se ha divido en dos grupos sobre la base del rendimiento escolar de 4to, 5to y 6to grado de escuelas públicas de Cuenca. En el Grupo 1, los niños de bajo rendimiento, con puntajes menores o iguales al percentil 25, lo que equivale a una calificación igual o menor a 17/20; y, en el Grupo 2, llamado también grupo de control, incluye a los niños de alto rendimiento escolar que pertenecen al percentil 75, cuya calificación es mayor o igual a 18,6/20. Criterios de exclusión: niños que, teniendo los criterios de inclusión, también tienen otras características como: discapacidad mental, que no completaron el test, que se retiraron o que no quisieron participar voluntariamente en el estudio.

Los dos grupos de estudio, ver Tabla 1, tanto de alto rendimiento como de bajo rendimiento no muestran diferencias significativas en cuanto a edad, años de escolaridad que han cursado, peso y talla, así como tampoco denotan diferencias significativas respecto a la edad del representante (padre, madre o algún familiar que represente legalmente al niño en la escuela), peso y talla según los percentiles de la OMS, las horas ofrecidas al juego, las horas dedicadas a ver la televisión, así como los años de experiencia de sus docentes dentro del campo educativo. 
Tabla 1. Características similares y diferentes de los grupos de alto y bajo rendimiento escolar.

\begin{tabular}{lccc}
\hline Variable & $\begin{array}{c}\text { Grupo 1: Bajo } \\
\text { rendimiento escolar } \\
(\mathrm{n}=391)\end{array}$ & $\begin{array}{c}\text { Grupo 2: Alto } \\
\text { rendimiento escolar } \\
(\mathrm{n}=318)\end{array}$ & $p$ \\
\hline Edad (años) & $10,5 \pm 0,9^{*}$ & $10,2 \pm 0,7$ & $\mathrm{~ns}$ \\
Grado & $6 \pm 1$ & $6 \pm 1$ & $\mathrm{~ns}$ \\
Peso (kg) & $29,75 \pm 14$ & $29,93 \pm 13$ & $\mathrm{~ns}$ \\
Talla (cm) & $115,3 \pm 49$ & $118,6 \pm 45$ & $\mathrm{~ns}$ \\
Edad del representante & $32 \pm 15$ & $31 \pm 16$ & $\mathrm{~ns}$ \\
Percentil de talla OMS & $41,3 \pm 28$ & $43,1 \pm 28$ & $\mathrm{~ns}$ \\
Percentil de peso OMS & $64,8 \pm 24$ & $65,4 \pm 24$ & $\mathrm{~ns}$ \\
Edad de padre al tener 1er hijo & $20 \pm 11$ & $22 \pm 10$ & 0,002 \\
Edad de madre al tener el ler hijo & $19 \pm 7$ & $21 \pm 7$ & 0,002 \\
Ingresos mensuales familiares & $459 \pm 950$ & $656 \pm 1506$ & 0,03 \\
Número de dormitorios & $2 \pm 1$ & $3 \pm 1$ & 0,01 \\
Horas dedicadas a jugar & $2 \pm 1$ & $2 \pm 1$ & $\mathrm{~ns}$ \\
Horas dedicadas a televisión & $1,68 \pm 0,9$ & $1,59 \pm 0,8$ & $\mathrm{~ns}$ \\
Experiencia del docente (años) & $7,5 \pm 6$ & $7,1 \pm 6$ & $\mathrm{~ns}$ \\
\hline Lejer & & &
\end{tabular}

Leyenda: $10,5 \pm 0,9^{*}=$ media y desviación estándar

Sin embargo, existen diferencias significativas respecto a la edad del padre y la madre al tener su primer hijo, los ingresos mensuales familiares y el número de dormitorios por familia. De este modo, la edad del padre al tener el primer hijo fue de $20 \pm 11$ años en el grupo de bajo rendimiento, mientras que, para el grupo de alto rendimiento, fue de $22 \pm 10$ años; la edad de la madre del grupo de bajo rendimiento al tener su primer hijo es de $19 \pm 7$ años a diferencia de la madre del grupo de alto rendimiento que es de $21 \pm 7$ años; ello significa que los padres de niños con alto rendimiento fueron mayores a aquellos cuyos hijos coinciden con un bajo rendimiento. Respecto a los ingresos familiares, se distingue que los ingresos mensuales familiares de los niños con bajo rendimiento son de $459 \pm 950$ dólares y de los niños con alto rendimiento fue de $656 \pm 1506$ dólares; lo cual prueba que los ingresos económicos fueron más altos en las familias cuyos niños obtuvieron un alto rendimiento en la escuela. Finalmente, se observa que los hijos de aquellas familias que tienen $2 \pm 1$ dormitorios en sus casas coinciden con hijos cuyo rendimiento es bajo con respecto a las familias que tienen $3 \pm 1$ dormitorio.

\subsection{Métodos}

El nivel de estudio es de carácter exploratorio pues es la primera vez que se miden factores de riesgo asociados al desempeño escolar en la educación fiscal de Cuenca; de tipo transversal pues se aplicaron encuestas en una sola ocasión tanto a docentes, padres de familia como a estudiantes de alto y bajo rendimiento; y el trabajo fue realizado en forma prospectiva pues se midió la relación según el rendimiento académico obtenido en clases, paralelamente a la aplicación de los instrumentos.

Para abarcar todas las variables de riesgo social, se diseñaron cuatro instrumentos. El primero fue la encuesta individual para niños, la misma que abarcó aspectos fisiológicos, necesidades cognitivas, necesidades emocionales y sociales. El siguiente instrumento fue diseñado para padres de familia quienes dieron cuenta de las oportunidades que tienen de desarrollarse los niños en aspectos fisiológicos, cognitivos, afectivos y sociales dentro del hogar. Un tercer instrumento estuvo dirigido a los profesores de los niños, quienes, llenaron un instrumento por cada niño evaluado sobre quien se preguntó de las necesidades fisiológicas, cognitivas, afectivas y sociales dentro de la escuela. Finalmente, el cuarto instrumento estuvo orientado a conocer las oportunidades que tiene cada establecimiento a partir de sus políticas institucionales para hacer frente al riesgo social.

\footnotetext{
${ }^{1}$ OMS: Organización Mundial de la Salud
} 
Para el análisis de comparación y contraste se utilizó el software estadístico SPSS Versión 20,0. Se presenta el valor de la media, la desviación estándar de las variables numéricas y la t de Student con el método de comparación. El nivel de significación de las diferencias entre los dos grupos se marca con el valor de $p$. Si el valor es de $p \leq 0,05$, la diferencia es tomada como estadísticamente significativa. Para el análisis de comparación de porcentajes -expresado como en número de casos y el porcentaje correspondiente- se utiliza el test de $\chi^{2}$. Se utiliza el valor de p para la caracterización de niveles de significación; si es $p \leq 0,05$, se toma como estadísticamente significativo.

Para identificar los factores de riesgo y protección se realizan tablas de contingencia $2 \times 2$ en las que se acomodan a las variables en las filas y a los eventos en columnas (bajo rendimiento o alto rendimiento). Se calcula el Odds Ratio el cual indica los factores de riesgo y protección que tienen los niños de bajo rendimiento respecto a los de alto rendimiento. Los intervalos de confianza al 95\% muestran que si un resultado de Odds Ratio es menor a 1, se considera factor de protección, mientras que, si resulta mayor a 1, se considera factor de riesgo. Estos factores de riesgo y protección son significativos al 5\%, por lo que, se toma el valor de $p$ igual o menor a 0,05 de la prueba Mantel Haenszel.

\section{RESULTADOS}

Los resultados de la encuesta a nivel de los estudiantes, padres, profesores y directores escolares se presenta respectivamente en las Tablas 2 a 6 .

Tabla 2. Factores de riesgo y protección de la encuesta aplicada a estudiantes de alto y bajo rendimiento.

\begin{tabular}{|c|c|c|c|c|c|c|}
\hline \multirow[b]{2}{*}{ Variable } & \multirow{2}{*}{$\begin{array}{l}\text { Grupo 1: Bajo } \\
\text { rendimiento } \\
\text { escolar } \\
(\mathrm{n}=391)\end{array}$} & \multirow{2}{*}{$\begin{array}{l}\text { Grupo 2: Alto } \\
\text { rendimiento } \\
\text { escolar } \\
(\mathrm{n}=318)\end{array}$} & \multicolumn{4}{|c|}{ Odds Ratio intervalo de confianza ( $95 \%$ ) } \\
\hline & & & $\begin{array}{l}\text { Odds } \\
\text { Ratio }\end{array}$ & $\begin{array}{l}\text { Límite } \\
\text { inferior }\end{array}$ & $\begin{array}{l}\text { Límite } \\
\text { superior }\end{array}$ & $p$ \\
\hline Sexo masculino* & $202(51,7 \%)$ & $110(34,6 \%)$ & 2,021 & 1,490 & 2,740 & $<0,0001$ \\
\hline No cenar* & $19(4,9 \%)$ & $1(0,3 \%)$ & 16,191 & 2,155 & 121,620 & $<0,0001$ \\
\hline $\begin{array}{l}\text { Problemas constantes de salud } \\
\text { (percepción del niño)* }\end{array}$ & $206(52,7 \%)$ & $129(40,6 \%)$ & 1,631 & 1,210 & 2,200 & 0,001 \\
\hline Padre con trabajo ${ }^{\&}$ & $338(86,4)$ & $301(94,7 \%)$ & 0,360 & 0,204 & 0,636 & 0,001 \\
\hline $\begin{array}{l}\text { Motivación de padres a asistir a } \\
\text { clases \& }^{\text {\& }}\end{array}$ & $346(88,5 \%)$ & $299(94 \%)$ & 0,489 & 0,280 & 0,854 & 0,016 \\
\hline $\begin{array}{l}\text { Problemas constantes de salud } \\
\text { (percepción de los padres)* }\end{array}$ & $49(12,5 \%)$ & $20(6,3 \%)$ & 2,135 & 1,241 & 3,673 & 0,006 \\
\hline $\begin{array}{l}\text { El padre o madre juega con su } \\
\text { hijo }\end{array}$ & $275(70,3 \%)$ & $243(76,4 \%)$ & 0,732 & 0,522 & 1,026 & 0,007 \\
\hline $\begin{array}{l}\text { Cuando los niños están en casa } \\
\text { algún adulto los cuida }{ }^{\&}\end{array}$ & $273(69,8 \%)$ & $255(80,2 \%)$ & 0,572 & 0,403 & 0,811 & 0,002 \\
\hline $\begin{array}{l}\text { Tiene en casa libros y juguetes de } \\
\text { acuerdo a la edad }{ }^{\&}\end{array}$ & $275(70,3 \%)$ & $276(86,8 \%)$ & 0,361 & 0,244 & 0,533 & $<0,0001$ \\
\hline $\begin{array}{l}\text { Le ayuda a su hijo facilitando } \\
\text { materiales \& }\end{array}$ & $205(52,4 \%)$ & $193(60,7 \%)$ & 0,714 & 0,529 & 0,964 & 0,028 \\
\hline $\begin{array}{l}\text { Apoyo de sus padres con nuevas } \\
\text { ideas \& }\end{array}$ & $194(49,6 \%)$ & $194(61 \%)$ & 0,629 & 0,466 & 0,850 & 0,003 \\
\hline
\end{tabular}

Leyenda: ${ }^{\&}=$ factor de protección; $*$ = factor de riesgo

En la Tabla 2, se demuestra que existen 4 factores de riesgo y 7 de protección que arroja la encuesta realizada a los alumnos. De este modo, se demuestra que los niños de sexo masculino en la muestra $(51,7 \%)$ son 2 veces más probables de tener bajo rendimiento que los del sexo femenino. No cenar constituye un factor de riesgo pues quienes no lo hacen tienen probabilidad de tener bajo 
rendimiento 16 veces más que los que sí lo hacen. Los problemas de salud, a decir del niño, son una vez más probables de generar bajo rendimiento en los niños que aquellos que no padecen problemas de salud; mientras que, los problemas de salud, a decir de los padres, son dos veces más probables de generar bajo rendimiento en sus hijos. Por otro lado, los factores de protección para asegurar que los niños obtengan un alto rendimiento son: el trabajo del padre de familia, la motivación que los padres dan a sus hijos para que éstos asistan a clases, el juego de los padres con sus hijos, la existencia de algún adulto que cuide de los niños cuando se encuentran en casa, la existencia de libros y juguetes de acuerdo a la edad en casa, así como el apoyo de los padres con nuevas ideas dentro del hogar.

Tabla 3. Factores de riesgo y de protección de la encuesta realizada a los padres.

\begin{tabular}{|c|c|c|c|c|c|c|}
\hline \multirow[b]{2}{*}{ Variables } & \multirow{2}{*}{$\begin{array}{l}\text { Grupo 1: Bajo } \\
\text { rendimiento } \\
\quad \text { escolar } \\
(\mathrm{n}=391)\end{array}$} & \multirow{2}{*}{$\begin{array}{l}\text { Grupo 2: Alto } \\
\text { rendimiento } \\
\text { escolar } \\
(\mathrm{n}=318)\end{array}$} & \multicolumn{4}{|c|}{ Odds Ratio intervalo de confianza $(95 \%)$} \\
\hline & & & $\begin{array}{l}\text { Odds } \\
\text { Ratio }\end{array}$ & $\begin{array}{l}\text { Límite } \\
\text { inferior }\end{array}$ & $\begin{array}{l}\text { Límite } \\
\text { superior }\end{array}$ & $p$ \\
\hline $\begin{array}{l}\text { Luego de clases: Apoyo con algún } \\
\text { deporte }^{\&}\end{array}$ & $82(21 \%)$ & $93(29,2 \%)$ & 0,642 & 0,456 & 0,905 & 0,011 \\
\hline $\begin{array}{l}\text { Luego de clases: Apoyo con tareas } \\
\text { escolares }{ }^{\&}\end{array}$ & $261(66,8 \%)$ & $235(73,9 \%)$ & 0,709 & 0,511 & 0,983 & 0,039 \\
\hline $\begin{array}{l}\text { Existe apoyo económico para el } \\
\text { vestido en la casa }^{\&}\end{array}$ & $223(57 \%)$ & $216(67,9 \%)$ & 0,627 & 0,460 & 0,854 & 0,003 \\
\hline $\begin{array}{l}\text { Existe apoyo económico para } \\
\text { salud en la casa }{ }^{\&}\end{array}$ & $271(69,3 \%)$ & $262(82,4 \%)$ & 0,483 & 0,337 & 0,692 & $<0,0001$ \\
\hline $\begin{array}{l}\text { Padres creen que el desempeño de } \\
\text { su hijo es muy bueno o bueno\& }\end{array}$ & $128(32,7 \%)$ & $246(77,4 \%)$ & 0,142 & 0,102 & 0,200 & $<0,0001$ \\
\hline $\begin{array}{l}\text { El hijo asiste en forma real a un } \\
\text { centro de tareas* }\end{array}$ & $30(7,7 \%)$ & $10(3,1 \%)$ & 2,560 & 1,231 & 5,320 & 0,012 \\
\hline $\begin{array}{l}\text { En casa se dedica tiempo para } \\
\text { conversar con la familia }{ }^{\&}\end{array}$ & $150(38,4 \%)$ & $175(55 \%)$ & 0,509 & 0,377 & 0,687 & $<0,0001$ \\
\hline Relaciones cordiales en familia ${ }^{\&}$ & $265(67,8 \%)$ & $266(83,6 \%)$ & 0,411 & 0,285 & 0,592 & $<0,0001$ \\
\hline $\begin{array}{l}\text { Los padres conocen a los amigos } \\
\text { de sus hijos }{ }^{\&}\end{array}$ & $326(83,4 \%)$ & $282(88,7 \%)$ & 0,640 & 0,413 & 0,991 & 0,046 \\
\hline $\begin{array}{l}\text { Personas que rodean al niño son } \\
\text { un modelo adecuado\& }\end{array}$ & $276(70,6 \%)$ & $267(84 \%)$ & 0,458 & 0,317 & 0,664 & 0,046 \\
\hline $\begin{array}{l}\text { El niño es independiente: } \\
\text { alimentos, baño, limpieza dental, } \\
\text { tareas }^{\&}\end{array}$ & $265(67,8 \%)$ & $254(79,9 \%)$ & 0,530 & 0,375 & 0,749 & 0,046 \\
\hline
\end{tabular}

En la Tabla 3 se encuentra únicamente un factor de riesgo y 9 factores de protección en la encuesta realizada a los padres de familia o representantes de los niños en la escuela. El único factor de riesgo identificado es la asistencia en forma real a un centro de tareas (es importante señalar que generalmente quienes asisten a un centro de tareas son niños con problemas de aprendizaje que requieren apoyo extra-curricular) tienen 1 probabilidad más de tener bajo rendimiento en la escuela que aquellos que no asisten a un centro de apoyo de tareas, sin embargo, bajo el criterio de necesidad extra-curricular no se puede considerar como un factor de riesgo. Por otro lado, este instrumento demuestra que existen factores de protección significativos como es el apoyo de los padres de familia con algún deporte después de clases, el apoyo con las tareas en casa, el apoyo económico para el vestido en la casa, el apoyo económico para salud en la casa, creer que el desempeño de los hijos es bueno, dedicar tiempo para conversar con la familia en casa, mantener relaciones cordiales dentro de la familia, conocer a los amigos de los hijos, ofrecer un modelo adecuado de parte de las personas que rodean al niño y el hecho de tener hijos que sean independientes en cuanto a alimentarse, bañarse, lavarse los dientes y hacer las tareas. 
Tabla 4. Factores de riesgo y de protección de la encuesta realizada a los profesores.

\begin{tabular}{|c|c|c|c|c|c|c|}
\hline \multirow[b]{2}{*}{ Variables } & \multirow{2}{*}{$\begin{array}{c}\text { Grupo 1: Bajo } \\
\text { rendimiento } \\
\text { escolar } \\
(\mathrm{n}=391)\end{array}$} & \multirow{2}{*}{$\begin{array}{l}\text { Grupo 2: Alto } \\
\text { rendimiento } \\
\text { escolar } \\
(\mathrm{n}=318)\end{array}$} & \multicolumn{4}{|c|}{ Odds Ratio Intervalo de confianza (95\%) } \\
\hline & & & $\begin{array}{l}\text { Odds } \\
\text { Ratio }\end{array}$ & $\begin{array}{l}\text { Límite } \\
\text { inferior }\end{array}$ & $\begin{array}{l}\text { Límite } \\
\text { superior }\end{array}$ & $p$ \\
\hline Trabajos $^{\&}$ & $83(21,2 \%)$ & $288(90,6 \%)$ & 0,028 & 0,018 & 0,044 & $<0,0001$ \\
\hline $\begin{array}{l}\text { Los trabajos no fueron cumplidos } \\
\text { apropiadamente* }\end{array}$ & $313(80,1 \%)$ & $26(8,2 \%)$ & 45,067 & 28,121 & 72,224 & $<0,0001$ \\
\hline $\begin{array}{l}\text { El niño en tareas hace más de lo } \\
\text { que se le pedía }{ }^{\&}\end{array}$ & $5(1,3 \%)$ & $82(25,8 \%)$ & 0,037 & 0,015 & 0,093 & $<0,0001$ \\
\hline Eran creativos ${ }^{\&}$ & $46(11,8 \%)$ & $157(49,4 \%)$ & 0,137 & 0,094 & 0,200 & $<0,0001$ \\
\hline Involucramiento alto en clase ${ }^{\&}$ & $30(7,7 \%)$ & $272(85,5 \%)$ & 0,014 & 0,009 & 0,023 & $<0,0001$ \\
\hline $\begin{array}{l}\text { Participación sobresaliente en } \\
\text { clase }^{\&}\end{array}$ & $74(18,9 \%)$ & $295(92,8 \%)$ & 0,018 & 0,011 & 0,030 & 0,017 \\
\hline Deberes sobresalientes ${ }^{\&}$ & 10( & 235 & 0,009 & 0,005 & 0,018 & $<0,0001$ \\
\hline Pruebas sobresalientes ${ }^{\&}$ & $3(0,8 \%)$ & $208(65,4 \%)$ & 0,004 & 0,001 & 0,013 & $<0,0001$ \\
\hline Otros aportes sobresalientes \& & $9(2,3 \%)$ & $198(62,3 \%)$ & 0,014 & 0,007 & 0,029 & $<0,0001$ \\
\hline Repetidor de año* & $19(4,9 \%)$ & 0 & 33,346 & 2,005 & 554,490 & $<0,0001$ \\
\hline Buena sociabilidad de & $197(50,4 \%)$ & $265(83,3 \%)$ & 0,203 & 0,142 & 0,290 & $<0,0001$ \\
\hline $\begin{array}{l}\text { Aspecto personal bien } \\
\text { presentado }^{\&}\end{array}$ & $203(51,9 \%)$ & $287(90,3 \%)$ & 0,117 & 0,077 & 0,178 & $<0,0001$ \\
\hline $\begin{array}{l}\text { Institución educativa: se } \\
\text { acostumbra rotar a profesores } \\
\text { cada año* }\end{array}$ & $350(89,5 \%)$ & $266(83,6 \%)$ & 1,669 & 1,076 & 2,589 & 0,022 \\
\hline $\begin{array}{l}\text { El profesor potencia al alumno } \\
\text { con desarrollo de Destrezas }{ }^{\&}\end{array}$ & $43(11 \%)$ & $53(16,8 \%$ & 0,615 & 0,399 & 0,948 & 0,028 \\
\hline $\begin{array}{l}\text { El profesor potencia al alumno } \\
\text { con desarrollo de Aspectos } \\
\text { Intelectuales \& }\end{array}$ & $129(33,1 \%)$ & $138(43,7 \%)$ & 0,638 & 0,469 & 0,866 & 0,004 \\
\hline $\begin{array}{l}\text { El profesor potencia al alumno } \\
\text { con desarrollo Social y Personal }^{\&}\end{array}$ & $82(21 \%)$ & $88(27,8 \%)$ & 0,690 & 0,488 & 0,975 & 0,035 \\
\hline Otros ${ }^{\&}$ & $302(77,4 \%)$ & $267(84.5 \%)$ & 0,630 & 0,428 & 0,927 & 0,019 \\
\hline
\end{tabular}

Los resultados obtenidos de la encuesta aplicada a los profesores muestran en la Tabla 4 que existen tres factores de riesgo y 14 factores de protección. Los factores de riesgo, en base a las respuestas de los profesores, implican a los niños cuyos trabajos que no fueron cumplidos apropiadamente de tener 45 veces más de probabilidad de obtener bajo rendimiento que aquellos que cumplen con sus trabajos apropiadamente. Otro factor de riesgo ocurre en los niños repetidores de año, en ellos se observa que tienen 33 veces más probabilidad de tener un bajo rendimiento que aquellos que no han repetido el año. Por último, el hecho de rotar a los docentes en los distintos años de básica anualmente es 1,7 veces más probable de generar estudiantes con bajo rendimiento. Respecto a los factores de protección existentes para que los niños obtengan un alto rendimiento, se observa el cumplimiento de trabajos, hacer tareas más allá de lo esperado por el docente, ser creativos, involucramiento en la clase, la participación sobresaliente en clase, el cumplimiento de deberes sobresalientes, las pruebas sobresalientes, otros aportes sobresalientes, la buena sociabilidad del alumno, el aspecto personal bien presentado, la potenciación del docente en el alumno sobre el desarrollo de destrezas, aspectos intelectuales, sociales e intelectuales, entre otros.

El único factor de riesgo significativo, hace referencia a bajo compromiso por parte de la comunidad educativa para alcanzar los objetivos como 1,9 veces más probable de generar estudiantes de bajo rendimiento que aquellos establecimientos que se comprometen en alcanzar los objetivos. La encuesta aplicada a los directores de los establecimientos estudiados ofrece tres factores de riesgo no significativos, a saber: ausentismo de los niños en clases, socialización de objetivos y metas en la comunidad educativa, así como la retroalimentación de profesores y actores educativos sobre las decisiones institucionales. Así también, existe un factor de protección como es la actuación del director con flexibilidad frente a la inasistencia de los alumnos a clase. Cabe señalar que también 
existen factores de protección no significativos, a saber la falta de políticas para disminuir el ausentismo y a nivel general la falta de políticas definidas.

Tabla 5. Factores de riesgo y de protección de la encuesta realizada a los directores de escuela.

\begin{tabular}{|c|c|c|c|c|c|c|}
\hline \multirow[b]{2}{*}{ Variables } & \multirow{2}{*}{$\begin{array}{l}\text { Grupo 1: Bajo } \\
\text { rendimiento } \\
\text { escolar } \\
(\mathrm{n}=391)\end{array}$} & \multirow{2}{*}{$\begin{array}{l}\text { Grupo 2: Alto } \\
\text { rendimiento } \\
\text { escolar } \\
(\mathrm{n}=318)\end{array}$} & \multicolumn{4}{|c|}{ Odds Ratio Intervalo de confianza (95\%) } \\
\hline & & & $\begin{array}{l}\text { Odds } \\
\text { Ratio }\end{array}$ & $\begin{array}{l}\text { Límite } \\
\text { inferior }\end{array}$ & $\begin{array}{l}\text { Límite } \\
\text { superior }\end{array}$ & $p$ \\
\hline Ausentismo alto en la escuela & $235(60,3 \%)$ & $178(56,3 \%)$ & 1,175 & 0,870 & 1,588 & 0,292 \\
\hline $\begin{array}{l}\text { El centro no tiene políticas para } \\
\text { disminuir el ausentismo }\end{array}$ & $122(32,3 \%)$ & $109(34,5 \%)$ & 0,865 & 0,631 & 1,185 & 0,366 \\
\hline $\begin{array}{l}\text { La institución no cuenta con } \\
\text { políticas definidas }\end{array}$ & $261(66,9 \%)$ & $230(72,8 \%)$ & 0,757 & 0,546 & 1,047 & 0,093 \\
\hline $\begin{array}{l}\text { Los objetivos y metas } \\
\text { institucionales son compartidos por } \\
\text { profesores, padres, alumnos y } \\
\text { administrativos }\end{array}$ & $390(100 \%)$ & $316(100 \%)$ & 1,234 & 0,024 & 62,354 & 0,916 \\
\hline $\begin{array}{l}\text { Considera que el compromiso de la } \\
\text { comunidad educativa por lograr los } \\
\text { objetivos es bajo* }\end{array}$ & $212(54,4 \%)$ & $121(38,3 \%)$ & 1,919 & 1,419 & 2,596 & $<0,001$ \\
\hline $\begin{array}{l}\text { Ante una decisión institucional, se } \\
\text { toma en cuenta los puntos de vista } \\
\text { de los profesores y otros actores } \\
\text { educativos }\end{array}$ & $390(100 \%)$ & $316(100 \%)$ & 1,234 & 0,024 & 62,354 & 0,916 \\
\hline $\begin{array}{l}\text { Procede frente a la inasistencia con } \\
\text { flexibilidad }^{\&}\end{array}$ & $369(94,6 \%)$ & $316(100 \%)$ & 0,027 & 0,002 & 0,450 & 0,012 \\
\hline
\end{tabular}

Leyenda: ${ }^{\&}=$ factor de protección; $*$ = factor de riesgo

Tabla 6. Importancia que los directores ofrecen para gestiones en el establecimiento.

\begin{tabular}{lccc}
\hline \multicolumn{1}{c}{ Variables cuantitativas } & $\begin{array}{c}\text { Grupo 1: } \\
\text { Bajo rendimiento } \\
\text { escolar }\end{array}$ & $\begin{array}{c}\text { Grupo 2: } \\
\text { Alto rendimiento } \\
\text { escolar }\end{array}$ & $p$ \\
\hline Coordinar la educación en relación a métodos de & $2,6 \pm 1,7$ & $2,9 \pm 2,0$ & 0,020 \\
enseñanza & $8,3 \pm 0,6$ & $8,4 \pm 0,7$ & 0,011 \\
Clima escolar positivo y seguro & $9,2 \pm 1,3$ & $8,9 \pm 1,6$ & 0,025 \\
Clima de respeto mutuo &
\end{tabular}

En la Tabla 6 se detallan las variables cuantitativas que corresponden a la encuesta a los 9 directores de las escuelas, los cuales detallan de 1 a 10 la importancia de las actividades citadas. Se pueden observar valores significativos en tres aspectos, como son coordinar la educación según el método de enseñanza adecuado, lo cual en el grupo de bajo rendimiento es de 2,6 $\pm 1,7$ y en el grupo de alto rendimiento 2,9 $\pm 2,0$; lo que significa que en el grupo de alto rendimiento habría una mejor coordinación que en los directores del grupo de bajo rendimiento. Igualmente el clima escolar positivo obtiene una calificación de $8,4 \pm 0,7$ en los de alto rendimiento, más alta que el resultado de bajo rendimiento que es de $8,3 \pm 0,6$. De su parte, el clima de respeto mutuo, muestra que hay un clima de respeto mutuo significativo en el grupo de bajo rendimiento de 9,2 $\pm 1,3$ en relación al 8,9 $\pm 1,6$ del grupo de alto rendimiento.

\section{DISCUSIÓN}

El principal objetivo de la presente investigación fue el determinar los factores de riesgo y protección que inciden en el rendimiento de los alumnos. Los factores de riesgo para el bajo desempeño escolar 
que emergieron en la investigación estaban relacionados con aspectos concernientes a los bajos niveles de ingresos económicos; problemas de salud de los niños y problemas de alimentación materializados en la falta de la cena; así como en aspectos educativos como la rotación anual de los profesores y la repitencia del año escolar. Estos factores ejercían su influencia además de una manera más intensa en los niños que en las niñas. Los factores de protección en relación con la dinámica familiar que se obtuvieron en nuestro estudio fueron: la conversación en casa, la motivación de los padres para que sus hijos asistan a la escuela, que los padres jueguen con los niños en casa, apoyo de padres con nuevas ideas, padre con trabajo, dentro del ámbito escolar el docente potencializador de su alumno, el involucramiento del niño en clases, el cumplimiento de tareas, el cuidado del aspecto personal del niño y la sociabilidad. En cuanto al análisis pormenorizado de los factores de riesgo, los problemas de salud se demuestran como factor importante de riesgo debido a que los niños con pérdida de salud deben faltar a horas escolares.

Este estudio demuestra también que los bajos ingresos económicos sí afectan el rendimiento escolar en los niños, lo que coincide con da Cunha y col. (2009), quien identifica a la inequidad social, al nivel de ingreso familiar y educación de los padres como influyentes para un mejor rendimiento académico. Igualmente, Jadue (1999) plantea que en las familias de niveles socioeconómicos bajos, no existe una motivación constante para que el niño vaya a la escuela, pues sus necesidades son otras, lo cual puede ser una razón para que los niños no se sientan alentados a obtener calificaciones altas o no le den la importancia necesaria al quehacer escolar. Por otro lado, un resultado de este estudio fue la vinculación entre rendimiento académico y trabajo paterno. El que la madre trabaje o no, no se ha mostrado un factor relevante en nuestro estudio para el rendimiento escolar; sin embargo, el que el padre lo haga sí ha emergido como un factor muy significativo para el rendimiento escolar de sus hijos. Este dato consideramos amerita una investigación más profunda.

Investigaciones que han analizado previamente las diferencias de rendimiento entre chicos y chicas indican que pueden existir diferentes factores de riesgo-protección para el desarrollo de bajo rendimiento académico y el fracaso para completar la educación para hombres y mujeres (Alspaugh, 2000). En términos de bajo rendimiento académico, la investigación indica que hay diferencias de género en los riesgos asociados con varones y mujeres (Whitney y col., 2010), pero estos no atañen específicamente a si es varón o mujer, sino más bien, a aspectos socio culturales relacionados con el género.

La repetición escolar significa un factor de riesgo que deviene de un historial de bajo rendimiento que parece no ser superado en los años escolares venideros. Al respecto, existen afirmaciones categóricas que ponen de manifiesto la casi nula mejora del desempeño escolar, así como la difícil adaptación personal de los niños repetidores (Shepard y Smith, 2008). Existen argumentos que, incluso, establecen una evolución negativa de casi todos los criterios afectivos y la infravaloración del alumno por parte del profesorado (Holmes y Mathews, 1984), posiblemente por todos los prejuicios y efectos cognitivos negativos de la repetición (Crahay, 2007). Nuestra investigación corrobora, en esta misma línea la escasa eficacia de la repetición de curso para la mejora del rendimiento académico de los estudiantes.

La rotación de los profesores en el sistema escolar contribuye negativamente al desempeño escolar. Al respecto, autores como López Yánez (2010), manifiestan que la dinámica de la alta rotación en la plantilla del profesorado que experimentan algunos centros educativos, supone mayor riesgo, si es que se considera que ello ocurre fundamentalmente en contextos difíciles. Es importante considerar que la rotación dificulta el desempeño profesional del docente pues no adquiere una total especialización en las materias de grado.

Las implicaciones alimentarias en el rendimiento académico son determinantes, algunas investigaciones indican que hay alimentos especialmente buenos para el cerebro, los que incluyen vegetales de hoja verde, salmón, frutos secos, carnes magras y frutas frescas (Connors, 1989). De igual forma, las vitaminas y los suplementos de minerales pueden reforzar el aprendizaje, la memoria y la inteligencia (Ostrander y Schroeder, 1991). Por ende, la falta de estos nutrientes termina por afectar las habilidades y destrezas de los educandos. En un estudio realizado en Kenia se encontró que los niños que tenían un déficit nutricional de dos desviaciones estándar por debajo de la media esperada tenían un rendimiento más bajo que aquellos que se encontraban en la media, Mukudi (2003). Cabe mencionar que la población de nuestro estudio estuvo en el peso y estatura adecuados. 
Sin embargo, se encontró que la falta de cena es un factor de riesgo asociado a bajo rendimiento, no solamente por el hecho biológico de la falta de alimentos previo al descanso, sino también por la diferencia de horas de ingesta desde el almuerzo hasta el desayuno. Como se conoce, la descarga energética del escolar es muy elevada, por lo que, para mantener el rendimiento adecuado, necesita alimentarse de forma correcta y regular. Ello implica que, al prescindir de la merienda estarían existiendo alrededor de $16 \mathrm{o} 17$ horas sin comer, en consecuencia, el no proveer el alimento necesario al cuerpo, pone en funcionamiento el autocontrol fisiológico del escolar para aprovecharlo en otros aspectos vitales. Adicionalmente, un estudio desarrollado por el Centro Nacional de Adicciones y Abuso de Sustancias de la Universidad de Columbia (CASA, 2009), establece una correlación entre la cena y la educación no formal del niño y/o adolescente. Según este estudio, al margen del aspecto fisiológico, la cena genera un encuentro familiar que reduce el riesgo a incurrir en adicciones, pues la cena es un espacio que permite a los padres e hijos desarrollarse en un modelo de relación interpersonal afín a la dinámica familiar. No obstante, se desconoce la existencia de un estudio que determine cuáles son las características del fenómeno de la ausencia de la cena en el desempeño escolar. Otros estudios realizados en 2 escuelas rurales de Perú encontraron resultados positivos del desayuno escolar en logros de memoria a corto plazo, aritmética y lectura, (Cueto y Chinen, 2008), también puede estar involucrada la atención (McEwan, 2012). En nuestro estudio la relevancia de rendimiento general, está en relación con la merienda.

Los factores que consideramos epifenómenos son: asistir a un centro a realizar tareas escolares extra clase -debido a que es una variable descriptiva del grupo de bajo rendimiento- y la no presentación de trabajos, según los estándares exigidos por el profesor -lo cual termina por afectar directamente las notas del rendimiento escolar del estudiante-. En relación a este hecho, Escudero (1982) cuestiona que el problema es determinar en qué medida estas notas son logros del rendimiento académico, pues la situación de fracaso escolar, al ser el resultado de un no suficiente éxito por parte del alumno en tareas escolares, o producto de trabajos que no se ajustan a los parámetros exigidos por el profesor, podrían no reflejar logros académicos, sino apreciaciones personales del docente.

Dentro de los factores de protección encontramos un buen número de variables, en relación con el apoyo e interés que padres y maestros pueden tener por sus hijos y alumnos en forma integral. Los resultados más significativos son la motivación de los padres a sus hijos para asistir a clases, cuidado de los niños en casa, apoyo con libros y juguetes acorde a la edad, apoyo con tareas en casa, apoyarles con nuevas ideas, percepción de los padres sobre el nivel de sus hijos, docentes que se interesan en el desarrollo global del niño. Todos estos factores están relacionados con la motivación familiar y del docente. Los estudios de Fan y Chen (2001) concluyen que, la participación de los padres en la escuela se asocia significativamente con el rendimiento académico. De la misma manera, un estudio realizado por Reynolds (1992) encuentra que la participación de padres y profesores de la escuela, aunque sólo modestamente correlacionados entre sí, ambos están asociados significativamente con los logros del niño en la escuela primaria temprana.

El apoyo con deportes, como era de esperar, constituye un importante aspecto protector pues concordando con Ramírez y col. (2004) la actividad física tiene una relación positiva con el rendimiento académico que se pueden notar en varias investigaciones, recogidas en su artículo de revisión teórica; aunque Feldman y Matjasko (2005) y McNeal y Ralph (1998) encuentran que actividades extracurriculares, no sólo el deporte, son un factor protector para el rendimiento académico. Otro factor protector es la buena sociabilidad, lo que concuerda con Fernández-Vilar y col. (2012), quien encuentra que el ajuste positivo del alumno evaluado por el profesor, actúa como predictor de casi todas las competencias académicas.

En relación al conocimiento actual de los autores, no se conoce un trabajo previo publicado que demuestre la importancia de la cena en el rendimiento escolar. La debilidad de esta investigación es que presenta un estudio transversal, frente al cual, se deberá plantear un estudio longitudinal a largo plazo. Con este trabajo se abre una línea de investigación social de los valores que acompañan a la cena en los niños de la ciudad de Cuenca. 


\section{CONCLUSIONES}

Este estudio develó como factores de riesgo importantes para el desempeño escolar, los problemas de salud de los niños: la falta de la cena, rotar a los profesores cada año, los ingresos económicos, la repetición del año escolar y el sexo masculino. Estos resultados deben analizarse en relación con otros factores, por ejemplo: los valores culturales que giran en torno al trabajo masculino, el que podría estar asociado en medio ecuatoriano, particularmente cuencano, con la cantidad de ingresos mayores del hombre en relación a la mujer, la estigmatización del niño repetidor de año y el valor de la cena. A partir de estos hallazgos puede proyectarse una guía de orientación para padres y docentes en búsqueda de una mejor calidad educativa y de vida para los niños que asisten a la educación pública del Ecuador.

\section{BIBLIOGRAFÍA}

Alspaugh, J.W., 2000. The effects of transition grade to high school, gender, and grade level upon dropout rates. Am. Second. Educ., 2-9.

Barnard, W.M., 2004. Parent involvement in elementary school and educational attainment. Child. Youth Serv. Rev., 26(1), 39-62.

Bonal, X., 2007. On global absences: Reflections on the failings in the education and poverty relationship in Latin America. Int. J. Educ. Dev., 27(1), 86-100.

Bowen, N., J-S. Lee, B. Weller, 2007. Social environmental risk and protection: A typology with implications for practice in elementary schools. Child. Sch., 229-242.

CASA, 2009. Annual Report. The National Center on Addiction and Substance Abuse at Columbia University. Descargado de http://www.casacolumbia.org/templates/Publications.aspx? articleid=598\&zoneid $=52$ el 08 de junio de 2012.

Crahay, M., 2007. Peut-on lutter contra l'échec scolaire? (3 éd.). Éditorial De Boeck, Bruxelles, Belgique.

Connors, C.K., 1989. Feeding the brain, how foods affect children. Editorial Perseus Books Group, London, UK, $280 \mathrm{pp}$.

Cueto, S., M. Chinen, 2008. Educational impact of a school breakfast programme in rural Peru. Int. J. Educ. Dev., 28(2), 132-148.

da Cunha, J.M., M.A. Jiménez, J.R. Rus Pérez, C. de Andrade, 2009. Social segregation and academic achievement in state-run elementary schools in the municipality of Campinas, Brazil. Geoforum, 40(5), 873-883.

Dunne, M., E.D. Ananga, 2013. Dropping out: Identity conflict in and out of school in Ghana. Int. J. Educ. Dev., 33(2), 196-205.

Escudero, J.M., 1982. El fracaso escolar hacia un modelo de análisis. En: Bartolomé, M. (Ed.), Modelos de investigación educativa. Editorial Universitat Barcelona, España, 17-75.

Fan, X., M. Chen, 2001. Parental involvement and students academic achievement: A meta-analysis. Educ. Psycol. Rev., 13(1), 1-22.

Feldman, A.F., J.L. Matjasko, 2005. The role of school-based extracurricular activities in adolescent development: A comprenhensive review and future directions. Rev. Educ. Res., 75(2), 159-210.

Fernández-Vilar, M., J. Carranza-Carnicero, M. Ato-García, 2012. Efecto del ajuste socioemocional en el rendimiento de las competencias académicas en el contexto escolar. Anales de Psicología, 28(3), 892-903.

González González, M.T., 2005. El absentismo y el abandono: una forma de exclusión escolar. Revista de Currículum y Formación de Profesorado, 9(1), 1-12. Descargado de http://www.redalyc.org/articulo.oa? id $=56790105$ el 12 de febrero de 2012. 
Holgado, D., I. Maya-Jariego, I. Ramos, J. Palacio, Ó. Oviedo-Trespalacios, V. Romero-Mendoza, J. Amari, 2014. Impact of child labor on academic performance: Evidence from the program "Edúcame Primero Colombia". Int. J. Educ. Dev., 34, 58-66.

Holmes, C., K. Mathews, 1984. The effects of nonpromotion on elementary and junior high schools pupils: A meta-analysis. Rev. Educ. Res., 54(2), 225-236.

INEC, 2011a. El Censo informa: Obtenido de Capítulo Educación. Descargado de http://www.inec.gob.ec/inec/index.php? option $=$ com_content \&view $=$ article \&id $=440 \% 3 \mathrm{Ael}$ censo-informa-capitulo-educacion \&catid=68\%3Aboletines\&Itemid=51\&lang=e el 16 de septiembre de 2011.

INEC, 2011b. Fascículo Provincial del Azuay. Instituto Ecuatoriano de Estadísticas y Censos. Descargado de http://www.ecuadorencifras.gob.ec/wp-content/descargas/Manulateral/Resultados-provinciales/azuay.pdf el 13 de enero de 2012.

INEC, 2013. Censo de población y vivienda 2010. Tablas de variables de educación. Descargado de http://redatam.inec.gob.ec/cgibin/RpWebEngine.exe/EasyCross? \&BASE $=$ CPV2010\&ITEM=IND ICEDU\&MAIN=WebServerMain.inl en octubre de 2013.

Jadue, G., 1997. Factores ambientales que afectan el rendimiento escolar de los niños provenientes de familias de bajo nivel socioeconómico y cultural. Estud. Pedagóg., 23, 75-80.

Jadue, G., 1999. Hacia una mayor permanencia de los niños en riesgo de bajo rendimiento y deserción. Estud. Pedagóg., 25, 83-90.

Kliksberg, B., 2009. América Latina: alto riesgo social. Descargado de http://elpais.com/diario/ 2009/06/24/opinion/1245794413_850215.html el 24 de junio de 2009.

Licove, J., 2009. Determinants of schooling for boys and girls in Nigeria under a policy of free primary education. Econ. Educ. Rev., 28(4), 474-484.

López Yánez, J., 2010. Liderazgo para sostener procesos de innovación en la escuela. Revista de Currículum y Formación del Profesorado, 14(1), 71-92. Descargado de http://www.ugr.es/ recfpro/ rev141ART4.pdf el 13 de enero de 2012.

Marchesi, Á., 2002. Cambios sociales y cambios educativos en Latinoamérica, 20 pp. Descargado de http://www.schwartzman.org.br/simon/delphi/pdf/marchesi.pdf el 12 de septiembre de 2012.

McEwan, P., 2012. The impact of Chile's school feeding program on education outcomes. Econ. Educ. Rev., 32, 122-139.

McNeal, J., B. Ralph, 1998. High school extracurricula activities: Closed structure and stratifying patterns of participation. J. Educ. Res., 91(3), 183-191.

MINEDUC, 2006. El Plan Decenal de Educación (2006-2015). Descargado de http://www.unicef.org/ ecuador/nacionalidades_y_pueblos_indigenas_web_Parte3.pdf en septiembre de 2012, 16 pp.

Mukudi, E., 2003. Nutrition status, education participation and school achivement among Kenyan middle-school children. Nutrition, 19(7-8), 612-616.

Ostrander, S., L. Schroeder, 1991. Supermemory: The Revolution. Editors Carroll \& Graf Publishers, New York, USA.

Ramírez, W., S. Vinaccia, G. Ramón, 2004. El impacto de la actividad física y el deporte sobre la salud, la cognición, la socialización y el rendimiento académico: Una revisión teórica. Revista de Estudios Sociales, 18, 67-75.

Reynolds, A., 1992. Comparing measures of parental involvement and their effects on academic achievement. Early Child. Res. Q., 7(3), 441-462.

Shepard, L., M.L. Smith, 2008. Synthesis of research on grade retention. Educational Leadership. Desargado de http://www.ascd.org/ASCD/pdf/journals/ed_lead/el_199005_shepard.pdf en marzo de 2010.

Whitney, S.D., L. Renner, T. Herrenkohl, 2010. Gender differences in risk protection profiles for low academic performances. J. Commun. Psycol., 435-455. 
Zeitlin, M., H. Ghassemi, M. Mansour, 1990. Positive deviance in child nutrition with emphasis on psycosocial and behavioural aspects and implications for development. The United Nations University, Tokyo, Japan, 153 pp. 